\title{
A 6-MONTH HISTOLOGIC AND HISTOMORPHOMETRIC ASSESSMENT OF XENOGRAFT OR XENOGRAFT COMBINED WITH PLASMA RICH IN GROWTH FACTORS VERSUS AUTOGENOUS BONE IN SINUS AUGMENTATION: A RANDOMIZED CONTROLLED TRIAL
}

\author{
Rehab Tarek Elsharkawy*, Nesreen Khairy ${ }^{* *}$ and Radwa Taher Elsharkawy ${ }^{* * *}$
}

\begin{abstract}
Purpose: The purpose of this study was to evaluate the effect of combining the PRGF to Xenograft on quality of the newly formed bone in open sinus lifting procedures after six months.

Patients and methods: Thirty maxillary sinuses were augmented in twenty five patients, 18 males and 7 females, who had less than $5 \mathrm{~mm}$ of bone height. Maxillary sinuses were randomly allocated into one of three groups; the study group I, where the sinus was augmented using autogenous bone taken from the patient's chin, group II, in which the sinus was augmented using Xenograft mixed with saline, group III, in which the sinus was augmented using Xenograft mixed with Platelets rich in growth factors (PRGF). After 6 months healing period, core biopsies were harvested and implant was simultaneously placed in the same site. Comparative microscopic and histomorphometric analysis were conducted and statistically analyzed. Implant loading was done after 6 months healing period.
\end{abstract}

Results: After 6 months, the results of this study showed that there was no adverse effect noticed in any case during the follow-up period. Histological findings showed that the addition of PRGF promotes bone maturation preceding the bovine grafted material alone. The histological stained specimens of group III revealed a picture more or less resembling group I (autogenous bone). The amount of the residual graft particles in the group I and III were found to be less than group II.

Conclusion: Combining PRGF to the xenograft improved the handling characteristics and the quality of the bone after 6 months in maxillary sinus augmentation procedure. Although PRGF combined to xenograft promoted bone maturation preceding the bovine grafted material alone, it did not prove to be superior to the autogenous bone which remains to be the gold standard for grafting.

KEY WORDS: Xenograft, Bovine bone, PRGF, Histomorphometric analysis, open sinus lift, maxillary sinus augmentation.

\footnotetext{
* Assistant Professor of Oral and Maxillofacial Surgery. Faculty of Dentistry, Cairo University

** Lecturer of Oral and Maxillofacial Surgery. Faculty of Dentistry, Cairo University

*** Lecturer of Ora Biology, Faculty of Oral and dental Medicine, Future University in Egypt.
} 


\section{INTRODUCTION}

Implant placement in the posterior maxillary area could be challenging due to anatomic limitations such as bone quality, inadequate vertical ridge height, or presence of undercuts. The vertical alveolar bone height is usually reduced in this area due to the maxillary sinus pneumatization as well as the postextraction bone resorption..$^{1,2}$

A number of sinus floor elevation techniques have been presented to augment bone height in the atrophic posterior maxillary area. The idea of sinus floor elevation was first designed and described by Tatum in 1970. The first published data on this subject matter were released by Boyne ad James in $1980 .^{3,4}$

The surgeries are based on trying to elevate the Schneiderian membrane and create a space below that membrane and the alveolar bone. This space is filled with a graft material to form new bone. Creating this space could be done using indirect or transcrestal approach or using a direct, lateral or open sinus lift approach. ${ }^{5,6}$

Many bone replacement materials have been tried in these techniques such as autogenous bone, allograft, xenograft, alloplastic bone, or combinations thereof. These different materials heal differently and mature over different time periods. ${ }^{7}$ Autogenous bone graft is considered to be the gold standard, but the fact that it requires another surgical site, the donor area, rendered it not widely accepted in clinical practice.

Autologous platelet concentrates have been used for sinus augmentations to shorten healing time, enhance soft and hard tissue healing, and improve bone quality when mixed with graft material. Platelet Rich Plasma PRP ${ }^{8,9}$ and Platelet Rich Fibrin PRF have been utilized in sinus augmentations. PRF has been tried to be used alone for sinus lifting ${ }^{10,11}$ and has been used also in combination with allograft or with xenografts. ${ }^{12,13}$
PRGF is relatively a recent addition to generation of autologous platelet concentrates. After its activation with calcium chloride, platelets release some growth factors and bioactive proteins, capable of modifying and improving healing of tissues. ${ }^{14}$ Different PRGF preparations were used in different treatment indications, both in dental as well as Medical fields. ${ }^{15}$ In 2004 Anitua et al, reported the use of PRGF to improve soft tissue heling ${ }^{16}$ and in 2006 he also tried using the liquid PRGF to bioactivate the surface of dental implants. ${ }^{17}$ A study was done by Anitua et al, in 2012 to evaluate the potential effects of PRGF when combined with osteoconductive bone material in comparison to bone only in sinus lifting in 5 cases. This preliminary study concluded that the PRGF may be a factor to reduce postoperative tissue inflammation, to increase new bone formation and to promote the bone vascularization. ${ }^{18}$

SmartBone is a xenogenic bone graft formed by a combination of bovine bone mineral and bioresorbable polymers with cell nutrients. This combination was done to improve the patient's cells growth into this xenograft providing osteogenesis, osteoconduction, and hopefully a better integration. Bioactive polymers give Smart bone great loading resistance, high volumetric stability as well as the polymers guard the bone from premature resorption. ${ }^{19}$

The aim of this prospective study was to evaluate whether combining the PRGF to Xenograft can improve quality of the newly formed bone in open sinus lifting procedures after six months.

\section{PATIENTS \& METHODS}

Thirty maxillary sinus augmentation procedures were done in male and female patients, who wanted to replace a missing posterior maxillary tooth with a dental implant. Selected cases were older than 18 years and they had to have a proper ridge width that is $6 \mathrm{~mm}$ or more and remaining alveolar bone height that is $5 \mathrm{~mm}$ or less to be eligible for inclusion in 
this study. Patients were not included if they have chronic infection or allergy in the maxillary sinus, pathologic condition in the area of surgery, uncontrolled systemic disease that might affect the surgery or bone healing or implant osseointegration, history of irradiation to the head and neck area; or if they are under chemotherapy or bisphosphonate treatment. Heavy smokers and pregnant and lactating females were also excluded from the study. All patients were selected from the out-patient clinic of the Oral and Maxillofacial Surgery department; Faculty of Dentistry, Cairo University.

Thorough clinical and radiographic examinations as well as evaluation of diagnostic casts, to assess the inter-arch relationship, were done for each patient. Eligible cases received detailed oral explanations about the nature of the study and the planned time frame till the prosthetic delivery. Patients, who approved the plan, had to sign a written informed consent form. After that, all patients underwent oral hygiene session to improve the oral environment before the augmentation procedure. Maxillary sinuses were randomly allocated into one of three groups; group I(control group) in which the sinus was augmented using autogenous bone taken from the patient's chin, group II ,in which the sinus was augmented using Xenograft mixed with saline, and group III, in which the sinus was augmented using Xenograft mixed with Platelets rich in growth factors (PRGF) .

Surgical phase: The sample randomization was achieved by the aid of a computer generated randomization table before the start of the surgeries. All patients received $2 \mathrm{~g}$ of Amoxicillin/ clavulanic acid(Augmentin $1 \mathrm{~g}$, Medical Union Pharmaceuticals (MUP) - ARE) and $8 \mathrm{mg}$ dexamethasone (SigmaTec Pharmaceutical Industries. ARE) 1 hour before surgery and they were asked to rinse the mouth with Chlorhexidine (Hexitol 0.12\%, the Arab drug company (ADCO) -ARE) for 1 minute. Surgeries were done under local anesthesia, except 2 patients, from the autogenous bone group, who were done under general anesthesia. A full-thickness mucoperiosteal flap was raised exposing the lateral bone wall of the antrum, where a bony window was created, with the inferior cut made $2 \mathrm{~mm}$ above the antral floor, using a diamond round bur on a hand-piece and Surgical motor (Implantmed drive unit, W\&H Dentalwerk, Bürmoos Salzburg, Austria). After completing the osteotomy, the Schneiderian membrane was gently separated from the surrounding bone using sinus lift curettes (ACE sinus lift kit, ACE surgical Supply Co., Inc. USA). After adequate elevation of the membrane, prepared bone (SmartBoneMicroships, IBI SA, MezzovicoVira, Switzerland) was packed into the sinus in small increments. The bony window was covered with a resorbable collagen membrane (Hypro-Sorb $\mathrm{M}$, bioimplon $\mathrm{GmbH}$, Germany) except in group 3, xenograft mixed with PRGF, where the window was covered by a membrane prepared from the PRGF, before the flap was readapted and closed.

After surgery, patients were instructed to take Amoxicillin/ clavulanic acid $1 \mathrm{~g}$ and $600 \mathrm{mg}$ ibuprofen (Brufen, Kahira Pharm. \& Chem. Ind. Co., ARE) twice a day and a systemic decongestant, loratadine / pseudoephedrine (Clarinase, Medical Union Pharmaceuticals (MUP), ARE) for 7 postoperative days. Patients were also instructed to use decongestant nasal drops OxymetazolineHCl (Afrin, Medical Union Pharmaceuticals (MUP) - ARE) 3 times a day and rinse their mouth with chlorhexidine twice daily till they come for suture removal 10 days after surgery. Patients were not allowed to wear removable prosthesis for 3 weeks after surgery and they were instructed to perform good oral hygiene and to avoid blowing their nose. Immediate panoramic x-ray was taken for each patient after surgery and follow up was done after 3 then 10 postoperative days.

For the control group I, bone harvesting started with vestibular incision in the mucosa 
between the canine teeth areas at least $1 \mathrm{~cm}$ beyond the mucogingival junction. A full thickness mucoperiosteal flap was reflected exposing the labial wall of the mandible at symphysis area. The osteotomies were performed with discs on a straight hand piece and completed with bone chisel and hammer. After delivery of the cortical graft, additional cancellous bone was collected from the chin using grooved chisel and finally the soft tissues were finally closed in two layers. The bone graft was milled using manual bone mill (ACE bone mill, ACE surgical Supply Co., Inc. USA) wetted in saline and packed into the elevated sinus.

For group II: the xenograft (1-2 mm particle size) (SmartBoneMicroships, IBI SA, MezzovicoVira, Switzerland) was wetted in saline.

For group III: the same xenograft was mixed with PRGF (Fig.1). The PRGF preparation started with collection of sixty $\mathrm{ml}$ of venous blood from the patient into 6 tubes ( $9 \mathrm{cc}$ each), containing sodium citrate anticoagulant. The PRGF was separated by centrifugation of the collected blood (80-1, Delta lab company, China) at $1800 \mathrm{rpm}$ for 8 minutes. The $1 \mathrm{~mL}$ plasma fraction located above the RBCS fraction, not including the buffy coat, was aspirated and transferred to 2 dishes where $50 \mu \mathrm{L}$ of calcium chloride were added for each $1 \mathrm{ml}$ of the preparation. The xenograft was then added and mixed with the preparation in one of the dishes and both dishes were incubated in a heated water path at $37^{\circ} \mathrm{C}$ for 40 minutes (Fig.2) (Anitua et al, 2012). ${ }^{18}$

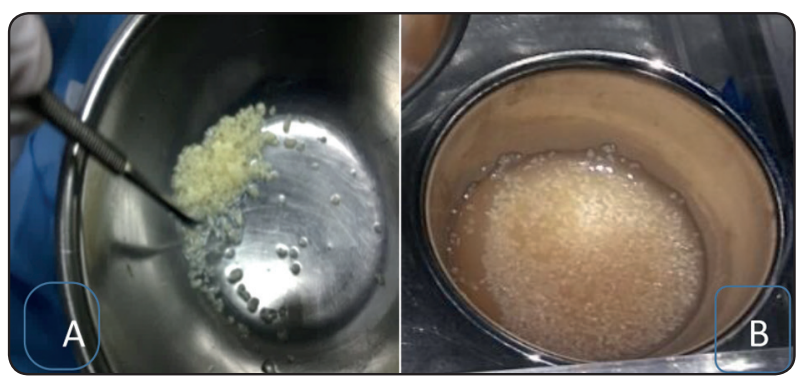

Fig. (1) A photograph showing Group II xenograft mixed with saline (A) and Group III, Xenograft in PRGF matrix (B).

\section{Sample acquisition and implant placement:}

Radiographic examination was done to evaluate the bone after 6 months healing period before samples from all augmented sinuses were obtained. A full thickness flap was elevated on the crest of the ridge where the implant osteotomy site preparation started with a trephine drill, $2.75 \mathrm{~mm}$ inside head diameter, $3.5 \mathrm{~mm}$ outside head diameter (ACE surgical Supply Co., Inc. USA). The trephine was used to collect bone core to a depth that contains both the original bone and the newly formed grafted bone, as planned from the preoperative radiograph. The biopsy specimens were fixed immediately in $10 \%$ neutral buffered formalin. After completing the osteotomy site preparation, the implant (MG InHex dental implants, MozoGrau, Spain) was inserted in position, covered with the cover screw and flap was sutured again. Finally, implants were loaded with the prosthesis after six months healing period.

\section{Histological examination}

After complete decalcification, specimens were processed, infiltrated in paraffin wax and embedded in the center of wax blocks. The embedded specimens were cut into $5 \mu \mathrm{m}$ thick sections. Specimens were sectioned and were stained by:

1. Hematoxylin and Eosin (H\&E) stain: After fixation, specimens were washed properly under running water, dehydrated by transferring through ascending concentrations of alcohol.

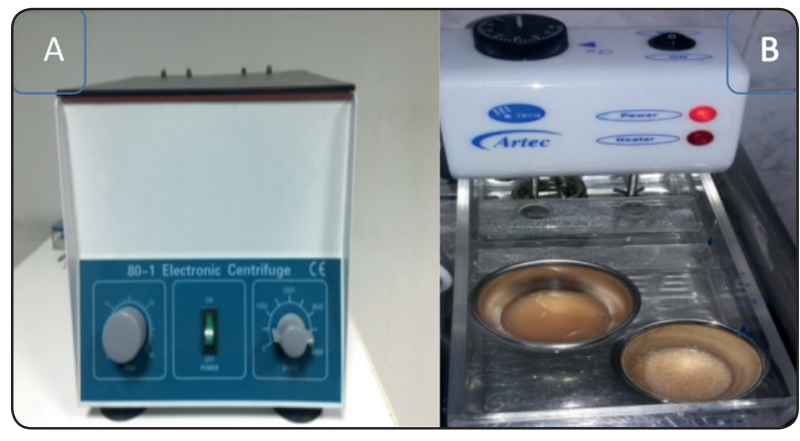

Fig. (2) Photograph of the centrifuge (A) and the hot water path containing a dish of preparation to be used as a membrane and the a second dish containing the PRGF mixed with Xenograft (B). 
The sections were stained by Hematoxylin and Eosin (H\&E) stain to be examined by light microscope (Trinocular microscope Olympus, BX46).

2. Masson trichrome special stain: It was used to detect areas of new bone formation as green color while old bone areas without new collagen formation appear red- dish in color when examined by light microscope. Paraffin sections were fastened to slides with Masson's gelatin. ${ }^{20}$

Histomorphometric analysis: The Histomorphometric analysis was carried out using the image analysis software (image J,1.41a, NIH, USA). The following parameters were evaluated the residual grafted particles in the H\&E stained sections of group II and III and the amount of collagen fibers in the Masson trichrome stained sections of all groups.

The collected data were statistically analyzed (Microsoft Excel AVERAGE, STDEV and T-TEST).

\section{RESULTS}

A total of thirty maxillary sinuses were grafted in 25 patients. Patients were 18 males $(72 \%)$ and 7 females (28\%). The age range of patients in this study was between 43 and 68 years (mean $55 \pm 7$ ). In 20 patients, one sinus was grafted and both sinuses were treated in the other 5 patients. At least one biopsy was harvested per sinus. All patients completed the follow up till loading the implants, with no attrition of the sample. All grafts and implants in the three groups integrated successfully. Postoperative pain and edema on the site of operation, whether the sinus or the chin in group A, were seen in all patients but there were no other significant postoperative complications.

\section{1-Microscopic assessments}

\section{Hematoxylin and Eosin (H\&E) stain}

The histological assessments of the specimens after 6 months healing period showed that bone formation in the three groups were evidence even though with differences in the quantity and quality of the formed bone. Group I: The specimens of the first group (autogenous bone graft) showed interconnected areas of complete bone formation. The newly formed bone appeared with the typical structure; lined by osteoblasts, normal sized osteocytes and well organized in their lacunae, parallel resting lines and tendency for forming the organized pattern of the Haversian system. Basophilic lines of demarcation of bone remodeling as well as resting lines were observed. The bone marrow tissue was well vascularized (Fig. 3).

Group II: The specimens of group II (bovine bone grafted material) showed islands of newly formed bone trabeculae, osteocytes revealed irregular arrangement and their lacunae were found relatively large. The bone marrow spaces are filled by fibro-cellular connective tissue rich in fibroblasts, undifferentiated mesenchymal cells and inflammatory cells. Remnants of the graft materials were observed accompanying the newly formed bone; they were easily identified because of their staining (Fig. 4).

Group III: In the specimens of the third group (PRGF+ xenograft), the newly formed bone trabeculae appeared well formed and lined by osteoblasts. Well vascularized marrow cavities, filled with fibroblasts and some osteoclasts on the peripheries were observed, indicating bone remodeling. No signs of inflammatory cells of fibrotic tissues were observed (Fig. 5).

\section{Masson trichrome stain}

The examination of the Masson trichrome stained sections of group I and group III showed collagen fibers of the newly formed bone matrices; indicated by the green color of the stain, they were mainly detected in areas showing bone remodeling, areas mineralized bone matrices were indicated with the red color (Figs. 6 and 7). In group II, most of collagen fibers were detected in the marrow spaces and surrounding the osteoblasts (osteoid tissue) (Fig.8). 
2-Histomorphometric analysis: The residual particles of the graft in the H\&E stained sections of group II were averaging $5.07 \pm 0.5 \%$ per area fraction while for group III was averaging $3.19 \pm$ $0.17 \%$ per area fraction, with calculated $\mathrm{P}$ value of 1.33. The amount of collagen fibers in the Masson trichrome stained sections of group I was found to

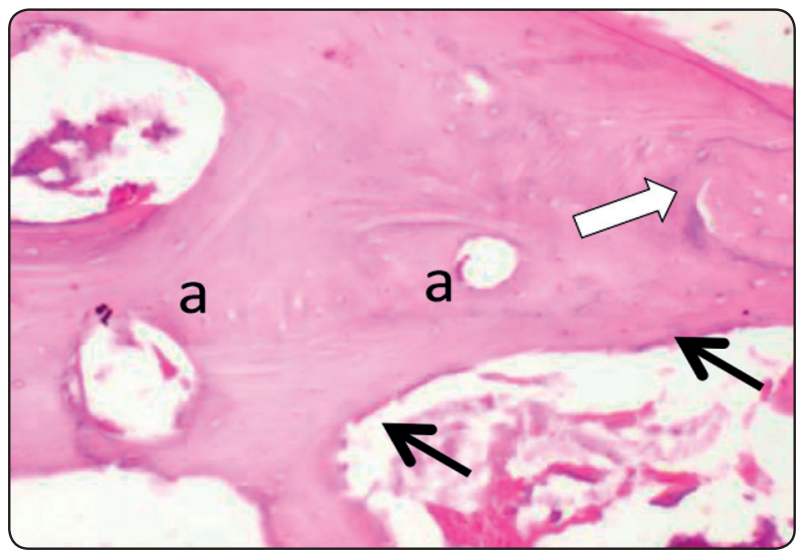

Fig. (3) Photomicrograph of the group I specimens showing well organized trabeculae, lined by osteoblasts (black arrows), normal sized osteocytes, haversian system formation(a), line of demarcation of bone remodeling(white arrow) and resting lines, well vascularized bone marrow tissue.(H\&E x100)

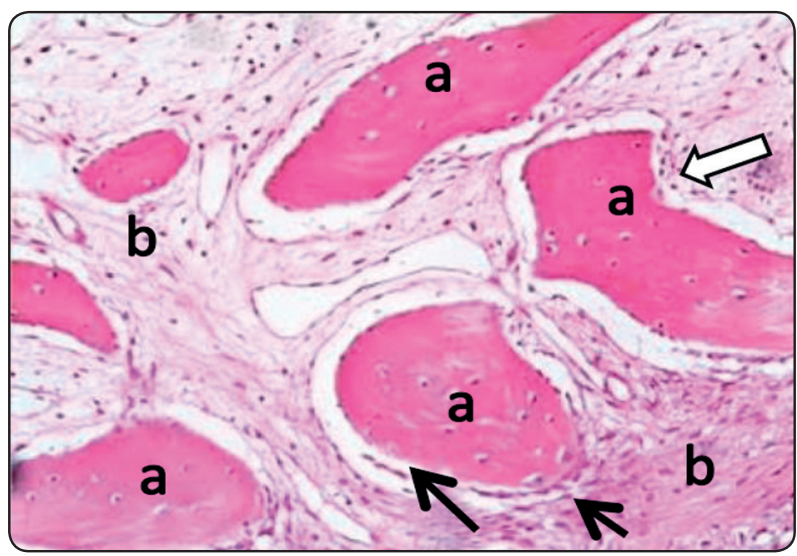

Fig. (5) Photomicrograph of the group III (PRGF + xenograft) specimens showing well formed the bone trabeculae (a), lined by osteoblasts( black arrows) and well vascularized marrow cavities, filled with fibroblasts (b) and osteoclastic activity(white arrow). (H\&E x100) be with a mean of $25.54 \% \pm 2.07 \%$ per area fraction, and for group II the mean was $18.98 \% \pm 0.53 \%$ per area fraction and for group III the mean was $33.43 \%$ $\pm 0.91 \%$ per area fraction. With a calculated $\mathrm{P}$ value between groups I and III of 0.0063 and between groups II and III of 1.4.

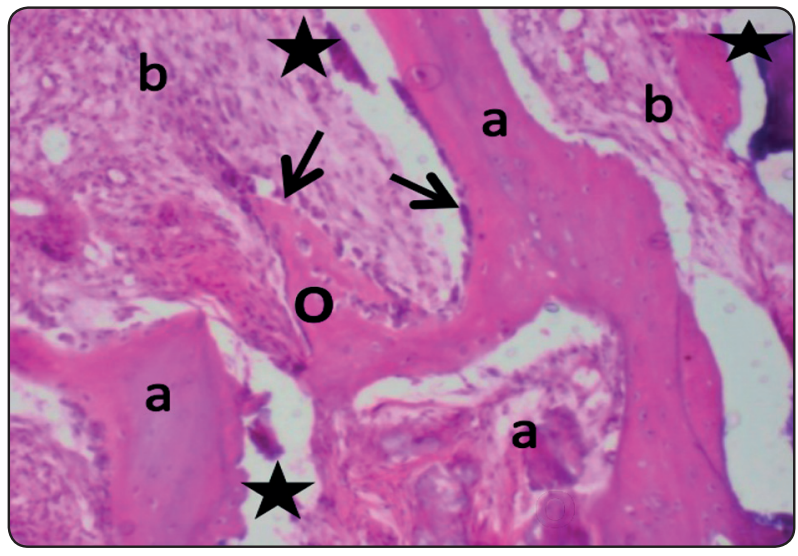

Fig. (4) Photomicrograph of the group II specimens showing islands of newly formed bone trabeculae (a), osteoblasts (black arrows), large osteocytes (o), the bone marrow spaces are filled by fibro-cellular connective tissue rich in fibroblasts, undifferentiated mesenchymal cells and inflammatory cells (b), remnants of the graft materials(stars). (H\&E x 100)

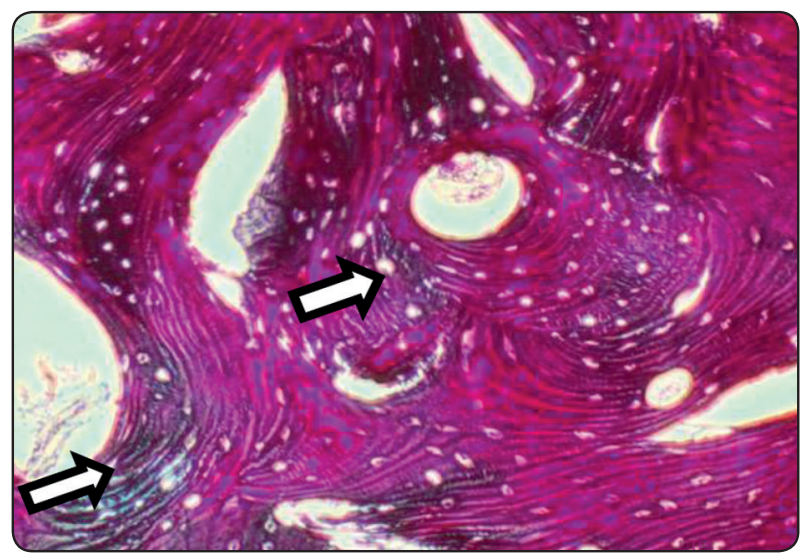

Fig. (6) Photomicrograph of the group I specimens showing some green areas containing collagen fibers of the newly formed bone matrices (white arrows) and areas of mineralized bone matrices indicated with red colors. (Masson trichrome $\mathrm{x} 100$ ) 


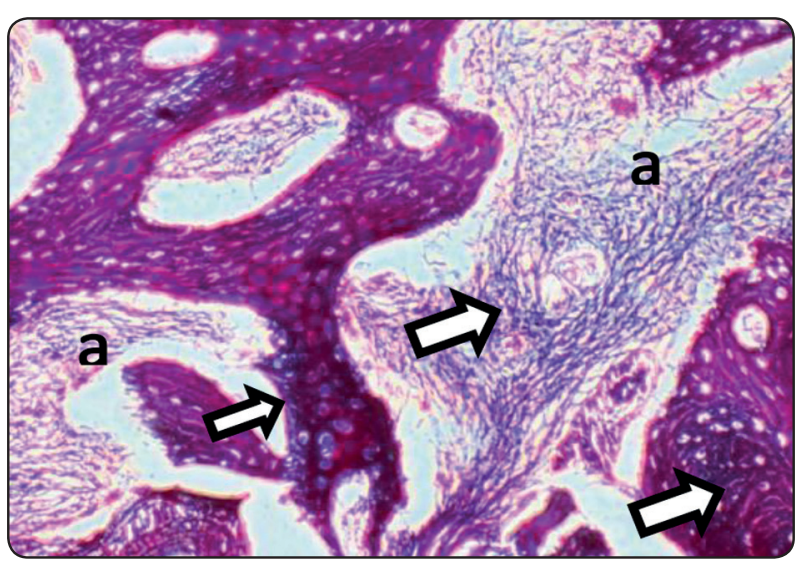

Fig. (7) Photomicrograph of the group II specimens showing collagen fibers were detected in the marrow spaces and surrounding the osteoblasts (osteoid tissue) (white arrow). (Masson trichrome x100)

\section{DISCUSSION}

Maxillary sinus floor lift became an important procedure for the rehabilitation of the atrophic posterior maxilla. Using grafts of different bone replacement materials became a standard technique to create the enough bone volume to accommodate dental implants. Different bone materials heal differently and mature over different time periods. The purpose of the present study was to evaluate the effect of mixing the PRGF with Xenogenic bone on healing and maturation of the graft material after sinus augmentation. That was done by comparing the histological results of PRGF-Xenograft to xenograft mixed with saline and to the gold standard of grafting material, the autogenous bone. From clinical point of view, the PRGF preparation was time consuming during surgery. But its addition to the Xenograft improved to a great extent the handling characteristics of the graft material. The PRGF acted like glue for the granular Xenograft, preventing their granules spreading and facilitating the administration and adaptation into the sinus. It also helped to avoid the uncontrolled displacement of the graft materials into the sinus cavity in the case of unnoticed Schneiderian membrane perforation. It

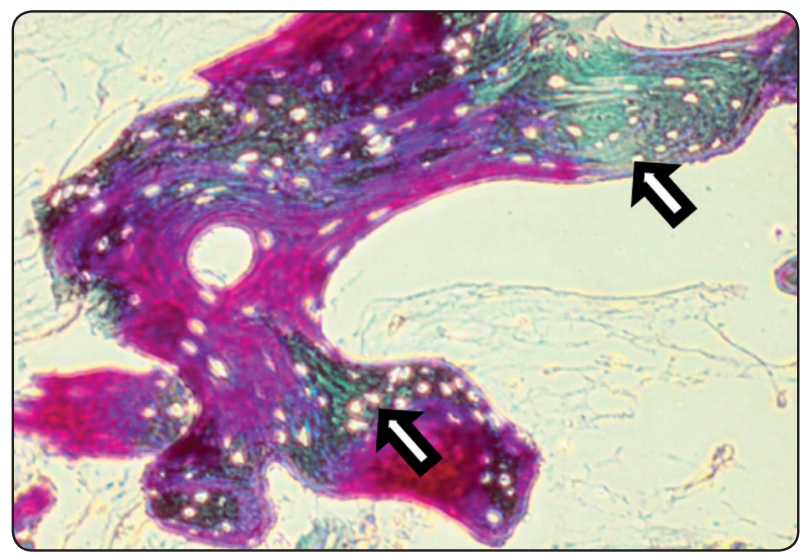

Fig. (8) Photomicrograph of the group III specimens showing mixed areas, some green areas containing collagen fibers of the newly formed bone matrices (white arrows) and areas of mineralized bone matrices indicated with red colors. (Masson trichrome x100)

also offered preparation of an autologous membrane that can be used to separate the graft material from the membrane as well as sealing of the osteotomy window before suturing. The use of this autologous membrane and not the collagen membrane decreased the overall cost of the operation.

The present study assessed the histological and histomorphometric differences between the following graft materials in maxillary sinus elevation procedure; autogenous bone grafts harvested from the symphysis, xenograft bone (smart bone) and PRGF + xenograft bone, group I, II and III consecutively. After 6 months healing period, the results showed that the usage of PRGF technology promotes bone maturation preceding the bovine grafted material alone, the histological stained specimens of group III revealed a picture more or less resembling group I (autogenous bone). The amount of the residual graft particles in the group I and III were found to be less than group II. They were found either in contact with the newly formed bone or accompanied by connective tissue and thereby, inflammatory cell infiltrations were encountered in all groups however it increased in group II. 
The usage of autogenous bone was proved to have a high osteogenic potential in comparison with other types of materials. Also, it has cells with bone induction capacity and does not induce any immunological reaction..$^{21}$ It was reported that Plasma rich in growth factors (PRGF) technology is an autologous plasma product rich in platelets which enables the local release of multiple growth factors and bioactive proteins that modify the processes of wound healing and tissue engineering. ${ }^{14,15}$ Platelets have the ability to release many growth factors and cytokines. Among these factors there are transforming growth factor beta (TGF- $\beta$ ), plateletderived growth factor (PDGF), vascular endothelial growth factor (VEGF), epithelial cell growth factor (EGF), basic fibroblast growth factor (bFGF) and platelet activating factor-4 (PAF-4). These growth factors known to play a significant role in bone regeneration and improvement of its vascularity. ${ }^{22,23}$

From results of the biomaterials that have been used in bone reconstruction, it is realized that there is a triad of components that coexist in the grafted site: newly formed bone, fragments of the biomaterial and fibrous connective tissue. The percentage of each depends on the type of material and type of study. ${ }^{24}$ Remnants of the bone grafted materials were noticed in group II more than the other experimental groups of the current study. It was reported that using platelet concentrates, such as PRGF, with the different bone grafts can enhance induction of osteoblasts and mesenchymal cells to the augmented area, which lead to accelerated cellular proliferation, matrix formation, and osteoid and collagen production. They cause an enhanced remodeling process, by accelerating the osteoclastic cells arrival and improving their invasion of the grafted particles. ${ }^{25,26}$

In group II of the present study irregular bone trabeculae with numerous entrapped osteocytes with large lacunae were evident. It was stated that the number of osteocytes depends on the rapidity of bone formation, so they increase in woven bone and decrease in lamellar bone. Osteocytes present in large lacunae during the early stages of their transformation from active osteoblasts to osteocytes as they have a well-developed Golgi apparatus for collagen storage, this also explained the greenish color found around the osteocytes in group I and III indicating stages of osteocyte maturation.

The residual particles of the graft in the $\mathrm{H} \& \mathrm{E}$ stained sections of group II were averaging 5.07 $\pm 0.5 \%$ per area fraction while for group III was averaging $3.19 \pm 0.17 \%$ per area fraction, with calculated $\mathrm{P}$ value of 1.33 which was statistically insignificant (with significant $P$ value $<0.05$ ). From the results the residual particles of the xenograft were found to be present at 6 months denoting ongoing creeping substitution process of the bone graft, thereby the grafted bone was not fully mature. However the PRGF group showed less amount of residual graft material thereby it accelerated the healing process of the graft compared with the usage of xenograft only, yet not similar to the autogenous bone specimens.

The amount of collagen fibers in the Masson trichrome stained sections of group I was found to be with a mean of $25.54 \% \pm 2.07 \%$ per area fraction, and for group II the mean was $18.98 \% \pm 0.53 \%$ per area fraction and for group III the mean was $33.43 \%$ $\pm 0.91 \%$ per area fraction. With a calculated $P$ value between groups I and III of statistically significant $\mathrm{P}$ value 0.0063 (with significant $P$ value $<0.05$ ) which confirmed the ongoing remodeling process of the newly formed bone of the PRGF group while the autogenous were found to be in a more mature state. The calculated P value between groups II and III was found to be non-significant with a value of 1.4 (with significant $\mathrm{P}$ value $<0.05$ ) thereby it was found that the PRGF with xenograft group was superior in terms of remodeling process in comparison to the xenograft alone. 


\section{CONCLUSION}

Combining PRGF to the xenograft material improved handling characteristics and quality of the bone after 6 months period in maxillary sinus augmentation procedure. Although PRGF combined to xenograft promoted bone maturation preceding the bovine grafted material alone, it did not prove to be superior to the autogenous bone which remains to be the gold standard for grafting.

\section{REFERENCES}

1. Pietrokoski J, Massler M. Alveolar ridge resorption following tooth extraction. J Prosthet Dent 1967; 17:21-7.

2. Sharan A, Madjar D. Maxillary Sinus Pneumatization Following Extractions: A Radiographic Study Int J Oral Maxillofac Implants 2008;23:48-56

3. Boyne P, James R. Grafting of the maxillary sinus floor with autogenous marrow and bone. J Oral Surgery.1980; 38: 613-616.

4. Tatum H Jr. Maxillary and sinus reconstructions. Dent Clin North Am 1986;30:207-29.

5. Misch, Carl E. Contemporary Implant Dentistry, 3rd ed. St Louis: Mosby, 934-6.

6. Summers RB, "The osteotome technique: part 3. -Less invasive methods of elevating the sinus floor," Compendium, vol. 15, no. 6, pp. 698-710, 1994.

7. Browaeys $\mathrm{H}$, Bouvry $\mathrm{P}$, and De Bruyn $\mathrm{H}, \mathrm{A}$ literature review on biomaterials in sinus augmentation procedures, Clinical Implant Dentistry and Related Research, vol. 9, no. 3, pp. 166- $177,2007$.

8. Boyapati L, Wang HL. The role of platelet-rich plasma in sinus augmentation: a critical review. Implant Dent. 2006;15:160-170.

9. Arora NS, Ramanayake T, Ren YF, Romanos GE. Plateletrich plasma in sinus augmentation procedures: a systematic literature review: Part II. Implant Dent. 2010;19:145-157

10. ToscanoN, and Holtzclaw D. Osteotome-mediated sinus floor elevation using only platelet-rich fibrin: an early report on 110 patients, Implant Dent. 2010; 19, 447- 456.

11. Diss A, Dohan DM,Mouhyi J, and Mahler P, Osteotome sinus floor elevation using Choukroun's platelet-rich fibrin as grafting material: a 1-year prospective pilot study with microthreaded implants," Oral Surg Oral Med Oral Pathol Oral RadiolEndod. 2008;. 105, 572-579.

12. Choukroun J, Diss A, Simonpieri A, Girard MO, Schoeffler C, Dohan SL, Dohan AJ, Mouhyi J, Dohan DM. Platelet-rich fibrin (PRF): A second-generation platelet concentrate. Part V: Histologic evaluations of PRF effects on bone allograft maturation in sinus lift. Oral Surg Oral Med Oral Pathol Oral RadiolEndod. 2006;101(3):299-303.

13. Zhang Y, Tangl S, Huber CD, Lin Y, Qiu L, Rausch-Fan X. Effects of Choukroun's platelet-rich fibrin on bone regeneration in combination with deproteinized bovine bone mineral in maxillary sinus augmentation: a histological and histomorphometric study.J Craniomaxillofac Surg. 2012 Jun;40(4):321-8

14. Anitua E, Sanchez M, Nurden AT, Nurden P, Orive G, Andia I. New insights into and novel applications for plateletrich fibrin therapies. Trends Biotechnol. 2006;24:227-234.

15. Anitua E, Sanchez M, Orive G, Andia I. The potential impact of the preparation rich in growth factors (PRGF) in different medical fields. Biomaterials. 2007; 28:4551-4560.

16. Anitua E, Andia I, Ardanza B, Nurden P, Nurden AT. Autologous platelets as a source of proteins for healing and tissue regeneration. ThrombHaemost. 2004; 91:4-15.

17. Anitua EA. Enhancement of osseointegration by generating a dynamic implant surface. J Oral Implantol. 2006; 32:72-76.

18. Anitua E, Prado R, Orive G. Bilateral sinus elevation evaluating plasma rich in growth factors technology: a report of five cases. Clin Implant Dent Relat Res. 2012; 14(1): 51-60

19. Abuelnaga MS, Elbokle NN, Khashaba MM. Evaluation of custom made xenogenic bone grafts in mandibular alveolar ridge augmentation versus particulate bone graft with titanium mesh. Research Gate. 2018, 9(2): 66-73.

20. J.D. Bancroft, M. Gamble (Eds.), Theory and practice of histological techniques (fifth ed.), Churchill Livingstone publishers, Philadelphia(2002)

21. Moy PK, Lundgren S, Holmes RE. Maxillary sinus augmentation: histomorphometric analysis of graft materials for maxillary sinus floor augmentation. J Oral Maxillofac Surg 1993; 51:857-62.

22. Weibrich G, Kleis WK, Hafner G, Hitzler WE, Wagner W. Comparison of platelet, leucocyte, and growth factor 
levels in point-of-care platelet-enriched plasma, prepared a modified Curasan kit, with preparation received from a local blood bank. Clin Oral Implants Res. 2003; 14(3):357-62.

23. Sanchez AR, Sheridan PJ, Kupp LI. Is platelet-rich plasma the perfect enhancement factor? A current review. Int J Oral Maxillofac Implants. 2003; 18(1):93-103.

24. Proussaefs, P. and Lozada, J. Use of titanium mesh for staged localized alveolar ridge augmentation: clinical and histologic-histomorphometric evaluation. J. Oral Implantol., 32(5):237-47, 2006.

25. Marx RE. Platelet-rich plasma: evidence to support its use. J Oral Maxillofac Surg 2004 Apr;62(4):489-96.

26. Paknejad M, Shayesteh YS, Yaghobee S, Shariat S, Dehghan M, Motahari P:Evaluation of the Effect of Plasma Rich in Growth Factors (PRGF) on Bone Regeneration. J Dent (Tehran). 2012; 9(1):59-67. 